\title{
Videoconference version of the Montreal Cognitive Assessment: normative data for Quebec-French people aged 50 years and older
}

\author{
Christine Gagnon ${ }^{1}$ (1) Miloudza Olmand ${ }^{1,2}$. Emma Gabrielle Dupuy ${ }^{1,3} \cdot$ Florent Besnier $^{1,3} \cdot$ Thomas Vincent $^{1}$. \\ Catherine-Alexandra Grégoire ${ }^{1}$ Marianne Lévesque ${ }^{1,2}$. Marie Payer ${ }^{1,4,5} \cdot$ Béatrice Bérubé $^{1,4}$. Juliana Breton ${ }^{1}$. \\ Catia Lecchino ${ }^{1,2}$. Nadia Bouabdallaoui ${ }^{1,3}$. Josep Iglesies-Grau ${ }^{1,3}$. Mathieu Gayda ${ }^{1,3} \cdot$ Paolo Vitali $^{6,7}$. Anil Nigam ${ }^{1,3}$. \\ Martin Juneau ${ }^{1,3} \cdot$ Carol Hudon $^{8,9} \cdot$ Louis Bherer $^{1,3,10}$
}

Received: 29 September 2021 / Accepted: 2 February 2022 / Published online: 18 February 2022

(c) The Author(s), under exclusive licence to Springer Nature Switzerland AG 2022

\begin{abstract}
Background The COVID-19 pandemic forced health professionals to rapidly develop and implement telepractice and remote assessments. Recent reviews appear to confirm the validity of a wide range of neuropsychological tests for teleneuropsychology and among these, the Montreal Cognitive Assessment (MoCA), a cognitive screening test widely used in clinical settings. The normative data specific to the context of videoconference administration is essential, particularly that consider sociodemographic characteristics.

Aims This study had for objective to develop French-Quebec normative data for videoconference-administration of the MoCA that consider sociodemographic characteristics.

Methods A total of 230 community-dwelling adults aged 50 years and older taking part in clinical trials completed the MoCA by videoconference. Regression analyses were run with sex, education, and age as predictors of the total MoCA scores, based on previously published norms. As an exploratory analysis, a second regression analysis was also run with cardiovascular disease as a predictor.

Results Regression analyses revealed that older age and lower education were associated with poorer total MoCA scores, for medium effect size $\left(p<0.001, R^{2}=0.17\right)$. Neither sex nor cardiovascular disease, were significant predictors in our analyses. For clinicians, a regression equation was proposed to calculate $Z$ scores.

Discussion This study provides normative data for the MoCA administered via videoconference in Quebec-French individuals aged 50 years and over.

Conclusions The present normative data will not only allow clinicians to continue to perform assessments remotely in this pandemic period but will also allow them to perform cognitive assessments to patients located in remote areas.
\end{abstract}

Keywords Montreal Cognitive Assessment $\cdot$ Teleneuropsychology $\cdot$ Telemedicine $\cdot$ Normative data

Christine Gagnon

christine.gagnon@icm-mhi.org

1 Centre ÉPIC and Research Center, Montreal Heart Institute, 5055 rue St Zotique Est, Montréal, QC H1T 1N6, Canada

2 Department of Psychology, Université de Montréal, Montréal, Canada

3 Department of Medicine, Université de Montréal, Montreal, Canada

4 Department of Psychology, Université du Québec à Montréal, Montréal, Canada
5 Research Center, Institut Universitaire en Santé Mentale de Montréal, Montréal, QC H1N 3M5, Canada

6 Research Centre for Studies on Aging, McGill University, Montréal, Canada

7 Department of Neurology and Neurosurgery, Faculty of Medicine, McGill University, Montreal, Canada

8 École de Psychologie, Université Laval, Québec, Canada

9 Centre de Recherche CERVO, Québec, Canada

10 Centre de Recherche de l'Institut Universitaire de Gériatrie de Montréal, Montreal, Canada 


\section{Introduction}

In March 2020, the world was put on hold because of the COVID-19 pandemic: several countries forced their populations to home confinement following the World Health Organisation's recommendation. These ongoing stayingat-home and social distancing measures to control virus transmission have for collateral effect to reduce in-person health services, among these, neuropsychological and cognitive assessments. Yet, these assessments are crucial to allow for the early detection of cognitive impairment, and consequently for early intervention [1]. Given that older adults are at higher risk of hospitalization and death from COVID-19 [2, 3], clinicians were required to rapidly implement teleneuropsychology in their practice, to maintain access to services. Although neuropsychologists and other health professionals did not traditionally use technology in their practice prior to the COVID-19 pandemic [4], it has been a catalyst for the development of remote assessments and has propelled telemedicine and teleneuropsychology to the forefront. Reflecting this phenomenon is the wave of recent publications having for topic remote assessments: whereas for the past 10 years, only one or two publications per year had the keyword "teleneuropsychology" on PubMed, in 2020, there were 22 publications, and in 2021, 36 articles have been published with this keyword. Overall, this trend in publications shows the growing interest and use of teleneuropsychology in clinical practice.

However, the shift from in-person cognitive assessments to off-site assessments, such as telephone or videoconference assessments, calls for the use of valid and sensitive tools that are able to reliably detect cognitive impairment. Furthermore, specific norms for videoconference administered tests are needed to adequately interpret scores. The Montreal Cognitive Assessment (MoCA) is a cognitive screening tool that is widely used by professionals in a clinical setting. Previous studies have shown that the MoCA is sensitive and can differentiate healthy older adults from adults with mild cognitive impairment and dementia [5]. Also, a previous study published by our group showed that the MoCA was useful to differentiate between different cardiovascular disease profiles [6]. Indeed, participants with stable heart failure obtained both statistically and clinically significant lower total MoCA scores than participants with low cardiovascular risk factors. However, to date, the MoCA has traditionally been used for in-person testing. In response to the COVID-19 crisis, Klil-Drori and collaborators [7] evaluated the use of a 20-point telephone version of the MoCA to establish a cut-off to determine normal cognitive functioning. The authors established a 17/20 cut-off, yet, they underlined that the Tele-MoCA is not sensitive enough to detect subtle cognitive impairments, such as mild cognitive impairment or dementia at its early stages [6]. Moreover, a telephone assessment does not allow observing the patient, which can add rich qualitative information to the assessment. Videoconference cognitive assessments could provide a compromise between in-person assessments and telephone assessments in the context of the COVID-19 pandemic, in addition to extend access to individuals living in remote areas.

Many recent publications have shown the validity of videoconference neuropsychological testing compared to face-to-face testing [8-10]. A review paper by Marra and collaborators [10] suggests that the remote administration of the MoCA shows good validity compared to its face-to-face administration. Indeed, the videoconference administered MoCA has been validated for its use in different populations. For instance, Chapman et al. [11] compared face-toface MoCA assessment to videoconference administration in post-stroke patients. The authors did not observe significant differences between performances in either testing modalities or perceived difficulty by participants. Similarly, DeYoung et al. [12] compared face-to-face and videoconference MoCA assessments with veterans living in rural areas. Here again, the authors showed that participants performed similarly in both conditions and suggested that the MoCA via telehealth is reliable. Importantly, this modality was acceptable for participants. Furthermore, videoconference administration of the MoCA increases access to patients located in remote areas. Finally, the Japanese version of the MoCA has also been recently validated for videoconference assessment [13]. Overall, the MoCA administered by videoconference appears to be a valid alternative to face-to-face assessment. However, in a recent letter to the editor, one of the co-authors of the MoCA underlines that non-standard administration of the MoCA, such as by videoconference, still does not have any normative data, which limits its interpretation [14]. Hence, the development of proper normative data for videoconference assessment is crucial to help clinicians interpret test scores. Moreover, it is crucial to develop adapted normative data that consider sociodemographic variables such as language, age, education, and sex. In this way, Larouche et al. [15] developed normative data for faceto-face administration of the MoCA in Quebec-French people. In their study, the authors developed regression equations allowing the calculation of $Z$ scores, considering sex, education, and age. The objective of the present project was to create normative data for videoconference administration of the MoCA in Quebec-French people considering sociodemographic characteristics of participants. Finally, as total MoCA scores have been found to differ according to cardiovascular disease profile [6], an exploratory objective of the present study was to determine whether the presence 
of cardiovascular disease could contribute to the regression equation.

\section{Methods}

\section{Participants}

Participants in the present study took part in two larger clinical trials, the COVEPIC trial [16] (clinicaltrials.org Clinical trial Identifier: NCT04635462) and the COVEPICARDIO trial (clinicaltrials.org Clinical trial Identifier: NCT04661189). Both trials have for objective to document the remote monitoring of home-based physical exercise alone and combined with cognitive training to promote cognitive and physical health of older adults during the COVID-19 pandemic period, in individuals with and without cardiovascular risk factors (COVEPIC) and in individuals with cardiovascular disease (COVEPICARDIO). The Montreal Heart Institute's research ethics board approved both studies (MHI 2019-2785), which were conducted in accordance with the Helsinki Declaration and in compliance with International Conference on Harmonization Good Clinical Practice (ICH-GCP). Participants provided consent prior to taking part in the study. The COVEPIC and COVEPICARDIO trials were designed to cope with the COVID19 pandemic context, with online tools implemented by the research team that allowed the study to be fully remote, with no on-site visit.

A total of 230 participants aged 50 and older were recruited, among these 127 participants with and without cardiovascular risk factors (COVEPIC: i.e., diabetes, obesity, dyslipidemia, hypertension, current smoking), and 103 participants with a diagnosis of cardiovascular disease (COVEPICARDIO: stable chronic systolic or diastolic heart failure, atrial fibrillation, documented atherosclerotic disease). Participants were included if they had access to the Internet, had access to a tablet (i.e., iPad or Android) or a computer, were able to do light to moderate aerobic exercise training and had no contraindication for exercise training. Exclusion criteria were the following: non-cardiopulmonary limitation to exercise (e.g., arthritis), severe exercise intolerance, respiratory disease (e.g., uncontrolled/severe asthma, COPD, COVID-19), Mini-Mental Scale Examination (MMSE) telephone version lower than 19/23 [17].

\section{Materials and procedure}

During a preliminary call, participants went through the consent form with the research assistant and provided oral consent before the subsequent transmission of written consent by email. Following consent, participants completed a telephone version of the Mini-Mental State Examination
[18] (MMSE) previously validated by Roccaforte and collaborators [17], the ALFI-MMSE. The version used in the COVEPIC and COVEPICARDIO trials was based on the ALFI-MMSE to the exception that one item was added, bringing the total to 23 points. Hence, our telephone-MMSE included the following items: orientation in time (/5), orientation in space (/5), 3-word repetition (/3), spelling WORLD in French backward (MONDE) (/5), recall of 3 words (/3), sentence repetition (/1), naming the item the participant is talking into (/1). The difference was that one item was added for space orientation: a question regarding the location of an important sightseeing place in Montreal ("In what area is located the Olympic Stadium?"). Furthermore, we used a more severe cut-off than in the original paper by Roccaforte et al. [17], which was 17 on 22. Indeed, our cut-off was 19 on 23 , to exclude participants with significant cognitive impairment. No participant was excluded for this reason.

On another day, participants completed a technology tutorial with a research assistant to ensure that Internet connection and tools (e.g., Zoom videoconference software) were functioning and mastered sufficiently prior to the remote cognitive assessment. On the following session, participants completed the French 7.1 version of the MoCA via videoconference by a trained psychometrician or a neuropsychologist. Because of the large scale of the study and because of the videoconference administration, slight adaptations were made. For the visuospatial/executive component, the short trail-making test version was performed orally. Via screen sharing, the examiner showed the short trail-making test and the example, and then asked the participant to tell where the arrow should go next while respecting ascending order and alphabetical order. Next, for the copy of the cube (1 point), the assessor showed the model to the participants via screen share, and participants then showed their drawing to the camera for scoring purposes. This was followed by the clock-drawing task (3 points). Participants were first instructed to avoid looking at their watch or at a clock and to draw a clock; standard MoCA instructions were then provided. Participants then showed their clock to the camera for scoring. Naming was assessed with the identification of three low-familiarity animal figures (a lion, a rhinoceros and a camel), for a total of three points. Here again, items were shown on the screen via screen sharing. Attention was assessed with a forward and backward digit span task ( 2 points), a letter tapping task (1 point) and a Serial 7 -subtraction task ( 3 points), for a total of six points. Language was assessed by the repetition of two complex sentences ( 2 points) and by a phonemic fluency task ( 1 point was awarded if $>11$ words were produced in one minute), for a total of three points. Abstraction was assessed with a two-item similarity task ( 2 points). Memory was assessed by a 5 min delayed recall of a five-word list learned previously (5 points). During the two readings of the list, participants 
were instructed to not write down the words. Finally, in the orientation subscale, standard administration was done, to the exception of one slight modification: the question "Now, tell me the name of this place, and which city it is in" was replaced by one question regarding an important sightseeing place in Québec ("In which city is located the Château Frontenac"). Participants were also asked to tell the assessor their location (5 points for orientation subscore). The total score on the MoCA was obtained by adding the scores on all these items, for a maximum total of 30 points. In the present study, the raw total score was used; thus, there was no onepoint correction for participants with $\leq 12$ years of education. However, participants were enquired on the total years of education they had completed, to include this variable in the regression equation. The upper limit of total years of education is 23 which is equivalent to the maximum number of years in the Quebec schooling system.

\section{Statistical analyses}

Power calculation based on Larouche et al.'s [15] regression equation $R^{2}$ value, with three predictors (age, sex and education), indicated that a sample size of 53 subjects should yield $95 \%$ power. Our variable of interest was the MoCA's total raw score on 30. Statistical analyses were run with MAC IBM SPSS version 27. First, descriptive statistics were run to examine normality, skewness, and kurtosis of data; all parameters were satisfactory based on Curran et al.'s criteria [19]. Extreme data were removed if below or over $\pm 3.29 \mathrm{SD}$, a cutoff suggested by Tabachnik and Fidell [20]. Accordingly, a participant was removed for this reason. Two other participants were removed from the sample because of missing data (1) and the diagnosis of a terminal disease received during the testing period (1), leaving us with a final total sample of 227 participants. Bivariate correlations were done among predictor variables (sex, age, and education) to assess multicollinearity and singularity. No sign of multicollinearity and singularity were noted. Since our exploratory goal was to determine if the presence of cardiovascular diseases could predict the MoCA's total score, a bivariate correlation was performed between the total raw score and the participant's categorization (with or without cardiovascular diseases). There was a trend towards significance between the two variables $(r=-0.122, p=0.066)$ justifying the inclusion of the presence of cardiovascular diseases in the regression model. Sex, age, and education were correlated to the total raw score of 30 to ensure that they each were sufficiently linked to our variable of interest prior to the regression analysis (all $p \mathrm{~s}<0.05$ ). The inclusion of these characteristics in our model was based on a previous publication of normative data for the paper-pencil version of the MoCA in French-Canadian older adults [15]. Curve estimations were run to examine the interactions between our continuous predictors (age and years of education) and a potential quadratic model. Linear regressions were then performed to determine the association between performance on the videoconference-administered MoCA and the sociodemographic characteristics: sex, age, years of education.

Following the linear regression, an inspection of residuals was performed to verify the assumptions of homoscedasticity and normality. No sign of heteroscedasticity or abnormality were found.

Then, to validate and verify the stability of the model, a linear regression with 5000 bootstrap samples and a 95\% confidence interval was performed. Assumptions of homoscedasticity and normality were respected.

\section{Results}

Participant characteristics and mean MoCA scores are presented in Table 1. The final sample comprised 227 participants, with a mean age of 65.98 years (range $=50-87$ years), $56.4 \%$ female participants and a mean education level of 16.69 years (range $=6-23$ years). The sample's MoCA subscores based on the presence or absence of cardiovascular disease are reported in the supplementary material (see Supplementary Table S1).

Results indicated that in our model, age, education and a quadratic term for education were predictive of the total MoCA score, $F(4222)=10.947, p<0.001, R^{2}=0.17$, adjusted $R^{2}=0.15$; corresponding to a medium effect size [21]. The best predictive model only included age $(\beta=-0.273, \mathrm{SE}=0.017, p<0.001)$, education $(\beta=1.257$,
Table 1 Participants' characteristics

\begin{tabular}{llll}
\hline & Total sample $n=227$ & $\begin{array}{l}\text { Healthy participants } \\
n=126\end{array}$ & $\begin{array}{l}\text { Cardiovascular } \\
\text { disease } n=101\end{array}$ \\
\hline Characteristics & & & \\
Age $M \pm S D$ & $66.0 \pm 7.9$ & $67.0 \pm 7.9$ & $65.1 \pm 7.9$ \\
Female (\%) & 56.4 & 77.8 & 29.7 \\
Education (years) $\mathrm{M} \pm \mathrm{SD}$ & $16.7 \pm 3.2$ & $17.0 \pm 2.7$ & $16.3 \pm 3.7$ \\
MoCA score $\mathrm{M} \pm \mathrm{SD}$ & $27.0 \pm 2.2$ & $27.2 \pm 2.1$ & $26.7 \pm 2.2$ \\
\hline
\end{tabular}

$M$ mean, $M o C A$ montreal cognitive assessment, $S D$ standard deviation 
$\mathrm{SE}=0.298, p=0.005)$ and the quadratic term for education (education $\mathrm{x}$ education; $\beta=-1.2049, \mathrm{SE}=0.009, p=0.019$ ) as predictors. Sex was not significant when entered in the model $(\beta=-0.075, \mathrm{SE}=0.277, p=0.240)$. As for our exploratory goal, the presence of cardiovascular disease was not a significant predictor in the model $(\beta=0.003$, $\mathrm{SE}=0.314, p=0.993$ ). Thus, this model suggests that only older age and lower education were associated with lower MoCA total scores. Our validation procedure with 5000 bootstraps yielded the same results with an $R^{2}$ of 0.17 . The validation regression coefficients, $p$ values and $95 \%$ confidence intervals are presented in Table 2. Based on the results from the regression model, the equation presented in Table 3 can be used to calculate the expected score of the MoCA given the age and years of education of a participant.

For this equation to be easily used in a clinical setting, the expected score is subtracted from the participant's real score and then, divided by the standard error of the estimate to obtain a $Z$ score. An excel file has been made available as supplemental material to calculate a $Z$ score (see Supplementary Spreadsheet S1).

\section{Discussion}

The present study had for objective to provide normative data for the MoCA administered by video conference in Quebec-French adults aged 50 years and older. In line with normative data previously published for the in-person version of the MoCA, the total score was significatively correlated to age, education and a quadratic term for education. However, sex did not remain a significant predictor when entered into our predictive model. Although these results are not in line with in-person MoCA norms in a Quebec-French population [14], they are in line with previous studies that did not observe a significant relationship between sex and the total MoCA score [22-24]. An explanation for the difference between our present results and the ones obtained by Larouche and collaborators [15] with the in-person normative data in Quebec-French individuals could be related to male/female ratio differences in both samples. Indeed,
Table 3 Information used to calculate a participant's MoCA Z scores according to their sociodemographic characteristics

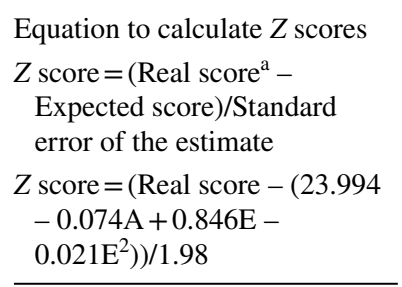

$A$ age in years, $E$ education in years, $M o C A$ montreal cognitive assessment

${ }^{\mathrm{a}}$ The real score represents the montreal cognitive assessment's total score

our sample comprised a more balanced male/female ratio (56\% female participants) compared to (67\% female participants) in their sample. Potential sex-related differences linked to education could explain this difference. In this way, $\mathrm{Lu}$ and collaborators [25] developed normative data in a Chinese sample and observed sex-related differences on the total MoCA score in participants with less than 5 years of education, with male participants performing better than female participants, whereas in participants with more than 5 years of education, sex differences were no longer present. Another explanation could be related to our participants' age-range. Indeed, our sample ranges from $50-87$ years of age, whereas in Larouche et al.'s sample, the range is from 40 to 98 years of age. This could potentially reflect sexrelated differences linked to generational differences since the recruitment of participants took place during distinct time periods.

Altogether, our regression model allowed us to develop an equation that will help clinicians to calculate $Z$ scores in their practice from the total MoCA score performed by videoconference. For instance, a 74-year-old woman with 11 years of education and a raw score of $27 / 30$ obtains a $Z$ score $=0.68$ with the present video conference-based assessment norms, whereas with the in-person administration derived equation provided by Larouche et al. [15] with the same demographic characteristics provides a $Z$ score $=0.56$. This suggests that the regression equation is
Table 2 Bootstrap validation procedure's regression coefficients

\begin{tabular}{lcrlcl}
\hline & \multicolumn{3}{l}{ Bootstrap $^{\mathrm{a}}$} & & \\
\cline { 2 - 6 } & Unstandardized B & \multicolumn{1}{l}{ Bias } & Standard Error & Sig (2-tailed) & 95\%CI \\
\hline Constant & 23.994 & 0.235 & 3.363 & $<0.001$ & $(18.14,31.45)$ \\
Age & -0.074 & 0.000 & 0.018 & $<0.001$ & $(-0.11,-0.04)$ \\
Education & 0.846 & -0.028 & 0.356 & 0.016 & $(0.03,1.45)$ \\
Education $^{2}$ & -0.021 & 0.001 & 0.010 & 0.035 & $(-0.04,0.002)$ \\
Sex & -0.326 & -0.008 & 0.275 & 0.235 & $(-0.88,0.19)$ \\
\hline
\end{tabular}

CI confidence interval

${ }^{a}$ Boostrap results are based on 5000 samples 
valid, provides similar results, as in both cases the patient obtains results that are in the average range. Moreover, as the equation derived from the videoconference administration of the MoCA does not include sex, contrarily to the in-person administration's norms, this reinforces the need for specific normative data for this administration context.

\section{Study Limitations}

Some limitations must however be considered when interpreting the present results. First, the present sample is highly educated (mean of 16.69 years), hence future studies with random sampling methods and including participants with more varied education levels may allow to avoid this potential bias. Moreover, from a clinical standpoint, our modification in the orientation subscale of the MoCA (replacing the question "Now, tell me the name of this place, and which city it is in" by a question regarding an important sightseeing place in Québec) tackles semantic memory more than orientation itself. This modification was made due to the context of video conferencing during confinement and the large scale of our studies. However, to our knowledge, the present study is the first to provide normative data for the video conference assessment of the MoCA and provides valuable information for clinicians. Another limitation is that the present study has not performed a validation or comparison with in-person total MoCA scores and videoconference total MoCA scores. Indeed, because of limitations linked to the COVID-19 pandemic, in-person testing could not be performed. However, the administration was in line with face-to-face administration instructions of the test and the adaptations were minor. Moreover, previous studies by Chapman et al. [11] and DeYoung et al. [12] have already shown significant correlations between in-person and videoconference administrations of the MoCA.

Among the strengths of the present study, none of the participants showed significant cognitive impairment, as confirmed by a telephone version of the MMSE based on the version by Roccaforte et al. [17]. Moreover, a technological tutorial was performed on a session prior to cognitive testing, so that participants were at ease with the videoconference application and connection process. This could possibly have reduced stress linked to the assessment. Indeed, previous studies have shown that the study context, such as a new and unknown environment (e.g., research center), could negatively impact older adults' memory performances and increase their cortisol levels [26].

\section{Conclusion and future directions}

To conclude, the present study provided normative data for Quebec-French adults aged 50 years and older for the
MoCA administered by videoconference. To our knowledge, this is the first study to provide such normative data, which is crucial for a test as widely used by clinicians. The present normative data will not only allow clinicians to continue to perform assessments remotely in the event of a future lockdown but will also allow performing cognitive screenings to patients located out of urban areas, where access to such services might be limited. Future studies should focus on providing normative data for other commonly administered neuropsychological tests administered by videoconference to comply with the growing interest and use of teleneuropsychology. Future studies validating the videoconference MoCA in clinical populations, such as individuals with mild cognitive impairment or dementia, could be relevant to validate the norms in these specific groups.

Supplementary Information The online version contains supplementary material available at https://doi.org/10.1007/s40520-022-02092-1.

Acknowledgements We would like to thank the EPIC Center of the Montreal Heart Institute and their members and patients for their active participation in the COVEPIC and COVEPICARDIO trials.

Author contributions CG: Conceptualization, methodology, writingoriginal draft preparation, project administration, formal analysis supervision; MO: conceptualization, writing-original draft preparation, formal analysis; EGD: investigation; FB: investigation; TV: investigation; C-AG: investigation; ML: investigation; MP: investigation; BB: investigation; JB: investigation; CL: investigation; NB: resources, review and editing; JI-G: investigation, review and editing; MG: investigation, resources; AN: Investigation, resources; PV: Investigation; AN: Investigation, resources; MJ: Investigation, resources; LB: writing-review and editing, supervision, funding acquisition, conceptualization.

Funding The Mirella and Lino Saputo Research Chair in Cardiovascular Health and the Prevention of Cognitive Decline from Université de Montréal at the Montreal Heart Institute supported this research.

Availability of data and material Data from the present study can be made available for sharing purposes from the principal investigator under reasonable request.

\section{Declarations}

Conflict of interest All authors report no conflict of interest.

Ethics approval The Montreal Heart Institute's research ethics board approved both studies (MHI 2019-2785), which were conducted in accordance with the Helsinki Declaration and in compliance with International Conference on Harmonization Good Clinical Practice (ICH-GCP).

Consent to participation All participants provided consent prior to taking part in the study.

Consent for publication All authors provided consent prior to submission of this manuscript. 


\section{References}

1. Livingston G, Huntley J, Sommerlad A et al (2020) Dementia prevention, intervention, and care: 2020 report of the lancet commission. The Lancet. https://doi.org/10.1016/S0140-6736(20) 30367-6

2. Morley JE, Vellas B (2020) Editorial: COVID-19 and older adults. J Nutr Health Aging 24:364-365. https://doi.org/10.1007/ s12603-020-1349-9

3. Shahid Z, Kalayanamitra R, McClafferty B et al (2020) COVID-19 and older adults: what we know. J Am Geriatr Soc 68:926-929. https://doi.org/10.1111/jgs.16472

4. Miller JB, Barr WB (2017) The technology crisis in neuropsychology. Arch Clin Neuropsychol 32:541-554

5. Nasreddine ZS, Phillips NA, Bédirian V et al (2005) The montreal cognitive assessment, MoCA: a brief screening tool for mild cognitive impairment. J Am Geriatr Soc 53:695-699. https://doi.org/ 10.1111/j.1532-5415.2005.53221.x

6. Gagnon C, Saillant K, Olmand M et al (2021) Performances on the montreal cognitive assessment along the cardiovascular disease continuum. Arch Clin Neuropsychol. https://doi.org/10.1093/ arclin/acab029

7. Klil-Drori S, Phillips N, Fernandez A et al (2021) Evaluation of a telephone version for the montreal cognitive assessment: establishing a cutoff for normative data from a cross-sectional study. J Geriatr Psychiatry Neurol. https://doi.org/10.1177/0891988721 1002640

8. Gnassounou R, Defontaines B, Denolle S et al (2021) Comparison of neuropsychological assessment by videoconference and face to face. J Int Neuropsychol Soc. https://doi.org/10.1017/S135561772 1000679

9. Hunter MB, Jenkins N, Dolan C et al (2021) Reliability of telephone and videoconference methods of cognitive assessment in older adults with and without dementia. J Alzheimers Dis 81:1625-1647. https://doi.org/10.3233/JAD-210088

10. Marra DE, Hamlet KM, Bauer RM et al (2020) Validity of teleneuropsychology for older adults in response to COVID-19: a systematic and critical review. Clin Neuropsychol 34:1411-1452

11. Chapman JE, Cadilhac DA, Gardner B et al (2019) Comparing face-to-face and videoconference completion of the montreal cognitive assessment (moca) in community-based survivors of stroke. J Telemed Telecare. https://doi.org/10.1177/1357633X19890788

12. DeYoung N, Shenal BV (2019) The reliability of the montreal cognitive assessment using telehealth in a rural setting with veterans. J Telemed Telecare 25:197-203

13. Iiboshi K, Yoshida K, Yamaoka Y et al (2020) A validation study of the remotely administered montreal cognitive assessment tool in the elderly japanese population. Telemed J E Health 26:920 928. https://doi.org/10.1089/tmj.2019.0134
14. Phillips NA, Chertkow H, Pichora-Fuller MK et al (2020) Special issues on using the montreal cognitive assessment for telemedicine assessment during COVID-19. J Am Geriatr Soc 68:942-944

15. Larouche E, Tremblay MP, Potvin O et al (2016) Normative data for the montreal cognitive assessment in middle-aged and elderly quebec-french people. Arch Clin Neuropsychol. https://doi.org/ 10.1093/arclin/acw076

16. Dupuy EG, Besnier F, Gagnon C et al (2021) COVEPIC (Cognitive and spOrt Virtual EPIC training) investigating the effects of home-based physical exercise and cognitive training on cognitive and physical functions in community-dwelling older adults: study protocol of a randomized single-blinded clinical trial. Trials 22:505-505. https://doi.org/10.1186/s13063-021-05476-2

17. Roccaforte WH, Burke WJ, Bayer BL et al (1992) Validation of a telephone version of the mini-mental state examination. J Am Geriatr Soc 40:697-702

18. Folstein MF, Folstein SE, McHugh PR (1975) "Mini-mental state": a practical method for grading the cognitive state of patients for the clinician. J Psychiatr Res 12:189-198

19. Curran PJ, West SG, Finch JF (1996) The robustness of test statistics to nonnormality and specification error in confirmatory factor analysis. Psychol Methods 1:16

20. Tabachnick BG, Fidell LS (eds) (2019) Using multivariate statistics. Pearson, Boston

21. Cohen J (1992) A power primer. Psychol Bull 112:155

22. Freitas S, Simoes MR, Alves L et al (2011) Montreal cognitive assessment (MoCA): normative study for the portuguese population. J Clin Exp Neuropsychol 33:989-996

23. Malek-Ahmadi M, Powell JJ, Belden CM et al (2015) Age- and education-adjusted normative data for the montreal cognitive assessment (MoCA) in older adults age 70-99. Neuropsychol Dev Cogn B Aging Neuropsychol Cogn 22:755-761. https://doi.org/ 10.1080/13825585.2015.1041449

24. Hayek M, Tarabey L, Abou-Mrad T et al (2020) Normative data for the montreal cognitive assessment in a lebanese older adult population. J Clin Neurosci 74:81-86

25. Lu J, Li D, Li F et al (2011) Montreal cognitive assessment in detecting cognitive impairment in Chinese elderly individuals: a population-based study. J Geriatr Psychiatry Neurol 24:184-190. https://doi.org/10.1177/0891988711422528

26. Sindi S, Juster R-P, Wan N et al (2012) Depressive symptoms, cortisol, and cognition during human aging: the role of negative aging perceptions. Stress 15:130-137. https://doi.org/10.3109/ 10253890.2011 .599047

Publisher's Note Springer Nature remains neutral with regard to jurisdictional claims in published maps and institutional affiliations. 